\title{
Ephéméride approchée de la comète Encke.
}

La comparaison de l'éphéméride de la comète Encke (A. N. 3962) avec l'observation, faite à Heidelberg

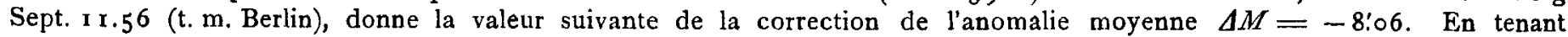
compte de cette correction, j'ai calculé l'éphéméride qui suit.

$\mathrm{o}^{\text {h }}$ t. m. Berlin.

\begin{tabular}{|c|c|c|c|c|c|c|c|c|c|}
\hline \multicolumn{2}{|c|}{ I 904} & \multicolumn{3}{|c|}{$\alpha$ app. } & \multicolumn{2}{|c|}{$\delta$ app. } & $\log r$ & $\log \Delta$ & t. d'ab. \\
\hline \multirow[t]{18}{*}{ Oct. } & I 4 & \multicolumn{3}{|c|}{$\mathrm{o}^{\mathrm{h}} 3^{8^{\mathrm{m}}} 46^{\mathrm{s}}$} & \multicolumn{2}{|c|}{$+28^{\circ}$ I $4: 5$} & 0.2248 & 9.8521 & $5^{m} 55^{s}$ \\
\hline & I 5 & & 35 & 3 & 28 & I 2.0 & 2215 & $845^{2}$ & 50 \\
\hline & 16 & & 31 & I 5 & 28 & 8.5 & 2 I 83 & $8_{3} 83$ & 44 \\
\hline & 17 & & 27 & 20 & 28 & 4. I & $2 \mathrm{I} 5^{\circ}$ & 8315 & 39 \\
\hline & I 8 & & 23 & 20 & 27 & 58.7 & 2 I 6 & 8248 & 33 \\
\hline & t 9 & & 19 & I 5 & 27 & $5^{2.5}$ & 2082 & 8182 & 28 \\
\hline & 20 & & I 5 & 4 & 27 & $45 \cdot 2$ & $204^{8}$ & $8 \times 17$ & 23 \\
\hline & $2 \mathrm{I}$ & & Io & 48 & 27 & $3^{6.9}$ & $20 \times 3$ & 8054 & $x 9$ \\
\hline & 22 & & 6 & 28 & 27 & $27 \cdot 7$ & 1977 & 7993 & I 4 \\
\hline & 23 & 0 & 2 & 3 & 27 & $17 \cdot 3$ & $194 \mathrm{I}$ & $793^{2}$ & Io \\
\hline & 24 & 23 & 57 & 34 & 27 & $5 \cdot 7$ & 1905 & 7872 & 5 \\
\hline & 25 & & 53 & 2 & 26 & $53 \cdot 1$ & 1868 & $781_{5}$ & \\
\hline & 26 & & $4^{8}$ & 27 & 26 & 39.4 & I $83 I$ & 7759 & $45^{8}$ \\
\hline & 27 & & 43 & 49 & 26 & 24.4 & I 793 & 7706 & 55 \\
\hline & 28 & & 39 & 8 & 26 & 8.2 & I 754 & 7654 & $5 I$ \\
\hline & 29 & & 34 & 25 & 25 & $5^{I} .1$ & I 714 & 7604 & $4^{8}$ \\
\hline & 30 & & 29 & 41 & 25 & $33 \cdot I$ & I 675 & 7555 & 44 \\
\hline & $3 I$ & & 24 & 55 & 25 & 13.7 & I 635 & 7507 & $4 \mathrm{I}$ \\
\hline Nov. & $\mathbf{I}$ & & 20 & 8 & 24 & 53.1 & I 594 & $746 I$ & $3^{8}$ \\
\hline & 2 & & I 5 & $2 \mathrm{I}$ & 24 & $3 \mathrm{I} \cdot 5$ & $155^{2}$ & 7420 & 35 \\
\hline & 3 & & IO & 34 & 24 & 8.6 & 1510 & 7380 & 33 \\
\hline & 4 & & 5 & $4^{8}$ & 23 & $45, I$ & 1467 & 7342 & 30 \\
\hline & 5 & 23 & 1 & 3 & 23 & 20.6 & 1424 & $73 \circ 5$ & 28 \\
\hline & 6 & 22 & $5^{6}$ & I9 & 22 & 55.1 & I 380 & 7270 & 25 \\
\hline & 7 & & $5 I$ & 37 & 22 & 28.7 & I 335 & 7237 & 24 \\
\hline & 8 & & 46 & 57 & 22 & I. 5 & I 289 & 7207 & 22 \\
\hline & 9 & 22 & 42 & I9 & $+2 I$ & 33.5 & 0.1243 & $9.7 \times 78$ & 420 \\
\hline
\end{tabular}

Poulkovo, le I 2 octobre 1904 .

\begin{tabular}{|c|c|c|c|c|c|c|c|c|}
\hline \multicolumn{2}{|c|}{1904} & \multicolumn{2}{|c|}{$\alpha$ app. } & \multicolumn{2}{|c|}{$\delta$ app. } & $\log r$ & $\log A$ & t. d'ab. \\
\hline Tov. & 9 & $22^{h} 42$ & ${ }^{n} I 9^{5}$ & $+21^{\circ}$ & $33 \cdot 5$ & 0.1243 & 9.7178 & $4^{\mathrm{m}} 20^{\mathrm{s}}$ \\
\hline & 10 & & 44 & 21 & 5.0 & 1195 & $7 \times 5 \mathrm{I}$ & I 8 \\
\hline & I I & & I I & 20 & 35.6 & 1147 & 7125 & I 7 \\
\hline & I 2 & 28 & 42 & 20 & 5.8 & 1098 & 7102 & 16 \\
\hline & I 3 & 24 & $x_{7}$ & 19 & $35 \cdot 3$ & 1048 & 7080 & 15 \\
\hline & 14 & 19 & 54 & I 9 & $4 \cdot 5$ & 0997 & 7059 & I 3 \\
\hline & 15 & I 5 & 34 & I 8 & 33.0 & 0946 & 7040 & I 2 \\
\hline & 16 & 11 & 18 & 18 & 1.2 & 0893 & $7 \circ 23$ & I I \\
\hline & r 7 & 7 & & I 7 & 29.0 & 0840 & 7008 & 10 \\
\hline & I 8 & $22 \quad 2$ & $5^{6}$ & r6 & $5^{6.4}$ & 0785 & 6993 & 10 \\
\hline & I 9 & 2 I $5^{8}$ & 49 & I 6 & 23.7 & $073^{\circ}$ & 6978 & 9 \\
\hline & 20 & 54 & 47 & I 5 & 50.9 & 0673 & 6965 & 8 \\
\hline & $2 \Upsilon$ & 50 & 47 & I 5 & I 7.8 & .615 & 6953 & 7 \\
\hline & 22 & 46 & 49 & I 4 & $44 \cdot 3$ & 0555 & 6942 & 7 \\
\hline & 23 & $4^{2}$ & 54 & I 4 & 10.6 & 0495 & 6931 & 6 \\
\hline & 24 & 39 & 4 & I3 & $37 \cdot 1$ & 0433 & 6921 & 6 \\
\hline & 25 & 35 & I 7 & I 3 & 3.6 & $037 \mathrm{I}$ & 6912 & 5 \\
\hline & 26 & $3^{x}$ & $3 I$ & I 2 & 29.7 & 0307 & 6904 & 5 \\
\hline & 27 & 27 & 47 & I I & 55.5 & 0242 & 6896 & 4 \\
\hline & 28 & 24 & & I I & $2 \mathrm{I} \cdot 3$ & 0175 & 6888 & 4 \\
\hline & 29 & 20 & $2 \mathrm{I}$ & IO & 46.9 & 0106 & 6880 & 3 \\
\hline & 30 & I 6 & 40 & Io & I 2.3 & 0.0036 & 6874 & 3 \\
\hline Déc. & I & I 3 & & 9 & $37 \cdot 5$ & $9 \cdot 9964$ & 6868 & 2 \\
\hline & 2 & & 20 & 9 & 2.4 & $9^{8} 9^{1}$ & 6862 & 2 \\
\hline & 3 & 5 & 40 & 8 & 27.0 & 9815 & 6855 & 2 \\
\hline & 4 & 2 I 2 & & 7 & $5 \cdot \cdot 3$ & 9738 & 6849 & 2 \\
\hline & 5 & $205^{8}$ & $2 I$ & +7 & 15.4 & 9.9659 & 9.6843 & 4 \\
\hline
\end{tabular}

M. Kaminsky.

(17) Thetis. Korrektion der Ephemeride (Berl. Jahrb. I906): 1904 Okt. x 3 - 4.99- - 7 "8. W. Luther.

Planet (460) [1900 FN]. Dem Planeten (460) [r900 FN] ist bei Gelegenheit der Astronomenversammlung in Lund der Name Scania gegeben worden.

Var. 159.1904 Pegasi. The Harvard photographs show that this object has existed for several years and is variable. E. C. Pickering.

Verkaufanzeige. The Telescopes and General Apparatus used for the production of the famous celestial photographs of the late Dr. Isaac Roberts, Starfield, Crowborough, Sussex, are to be sold. Also the Observatory, House, and Grounds (about $4 \frac{1}{2}$ acres in extent). Delightfully situated, 800 feet above sea-level, with magnificent views. The whole to be disposed of as it stands; or the Telescopes and Apparatus may be sold separately. On view at any time; and further particulars gladly supplied on application. W. S. Franks.

Berichtigungen zu Nr. 395 I Bd. 165 p. 237 Z. 6 v. u. statt: I.4803 lies: I.4883.

$$
\begin{aligned}
& \gg \gg 395^{2} \gg \gg \gg 243 \text { Z. } 3 \text { v.u. statt: } 5^{\mathrm{d}} 8^{\mathrm{h}} 47^{\mathrm{m}} 39^{3} \cdot 979 \text { lies: } 5^{d} 8^{\mathrm{h}} 47^{\mathrm{m}} 39^{8} \cdot{ }^{9} 8_{4} \text {. }
\end{aligned}
$$

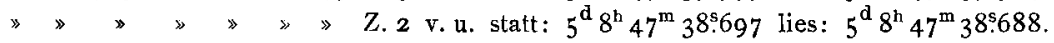

$$
\begin{aligned}
& \text { " } \gg \gg \gg, Z, \text { I v. u. statt: Is.277 lies: Is.296. }
\end{aligned}
$$

Inhalt zu Nr. 3972-73. F. Küstner. Spektrographische Beobachtungen am Bonner Refraktor. I77. - F. Palisa. Beobachtungen von kleinen Planeten auf der k. k. Sternwarte in Wien. 205. - M. Kaminsky. Ephéméride approchée de la comète Encke. 207. Kleine Mitteilungen. 207. - Verkaufsanzeige. 207. - Berichtigungen. 207. 